\title{
Analysis of Enoyl-Coenzyme A Hydratase Activity and Its Stereospecificity Using High-Performance Liquid Chromatography Equipped with Chiral Separation Column
}

\author{
Shirou Tsuchida* , Koutarou Kawamoto, Kana Nunome, Naoya Hamaue, \\ Teruki Yoshimura, Takashi Aoki and Takao Kurosawa \\ School of Pharmaceutical Sciences, Health Sciences University of Hokkaido (1757 Kanazawa, Tobetsu-cho, Ishikari-gun, Hokkaido 061-0293, \\ JAPAN)
}

\begin{abstract}
Enoyl-coenzyme A (CoA) hydratase catalyzes the hydration of trans-2-enoyl-CoA to yield 3-hydroxyacyl-CoA during fatty acid degradation ( $\beta$-oxidation). Although much research has focused on the stereospecificities of 2-enoyl-CoA hydratases, a direct quantification of the production of $3(R)$ - and $3(S)$ hydroxyacyl-CoA has not yet been established. Therefore, we developed a method of concurrently quantifying 3(R)- and 3(S)-hydroxyacyl-CoA using high-performance liquid chromatography (HPLC) equipped with a chiral separation column. The optimized conditions for the separation of $3(R)$-, $3(S)$ hydroxyhexadecanoyl-CoA and trans-2-hexadecenoyl-CoA, were determined to be as follows: mobile phase of 35/65 (v/v) of $50 \mathrm{mM}$ phosphate buffer (pH 5.0)/methanol; flow rate of $0.5 \mathrm{~mL} / \mathrm{min}$; detection at $260 \mathrm{~nm}$; and column temperature of $25^{\circ} \mathrm{C}$. This method was applied to subcellular fractions of rat liver; the results directly confirmed that $3(S)$-hydroxyhexadecanoyl-CoA is the dominant product obtained from the heatstable enoyl-CoA hydratase-catalyzed reaction of trans-2-hexadecenoyl-CoA. Finally, the stereospecificities of L-bifunctional protein (L-BP) and D-bifunctional protein (D-BP) were reinvestigated using this method, and it was confirmed that L- and D-BP yielded $3(S)$ - and $3(R)$-hydroxyhexadecanoyl-CoA were yielded from trans-2-hexadecenoyl-CoA, respectively. $3(R)$-Hydroxyacyl-CoA is a peroxisomal $\beta$-oxidation-specific intermediate. Therefore, this method is potentially useful not only studies regarding the stereochemistry of enoyl-CoA hydratase but also for the diagnosis of diseases caused by defects of peroxisomal enoyl-CoA hydratase.
\end{abstract}

Key words: chiral separation, enoyl-CoA hydratase, HPLC, 3-hydroxyacyl-CoA, peroxisomal $\beta$-oxidation

\section{INTRODUCTION}

Mammalian fatty acid degradation, known as $\beta$-oxidation, occurs not only in mitochondria but also in peroxisomes. Both systems involve the following four enzymatic reactions: dehydrogenation by acyl-coenzyme A (CoA) dehydrogenase (in mitochondria) or acyl-CoA oxidase(in peroxisomes), hydration by enoyl-CoA hydratase, oxidation by 3-hydroxyacyl-CoA dehydrogenase and thiolytic cleavage by 3-ketoacyl-CoA thiolase ${ }^{1-3)}$. As the molecular speciation and specificities of these enzymes differ between mitochondria and peroxisomes, it is not clear how this convergence in their reactivity occurs. Mitochondrial $\beta$-oxidation is known to produce energy for vital activities, whereas peroxisomal $\beta$-oxidation facilitates the degradation of very long-chain fatty acids $^{4)}$, branched-chain fatty acids ${ }^{5,6)}$, and the biosynthesis of bile acids ${ }^{7}$, among others. Accordingly, peroxisomal $\beta$-oxidation is essential for life. In fact, a disorder of this system, such as a deficiency of peroxisomes or the lack of one or more relevant enzymes, causes serious diseases $^{8-15)}$. Many researchers have studied the peroxisomal $\beta$-oxidation enzymes, discovering that two types of enoyl-CoA hydratases act as multifunctional proteins. These are the L-bifunctional protein (L-BP), also known as 2-enoyl-CoA hydratase/L-3-hydroxyacyl-CoA dehydrogenase, multifunctional enzyme 1 (MFE-1), or multifunctional protein 1 (MFP-1), and the D-bifunctional protein (D-BP), also known as D-3-hydroxyacyl-CoA dehydratase/D-3hydroxyacyl-CoA dehydrogenase, multifunctional enzyme 2 (MFE-2), or multifunctional protein $2(\mathrm{MFP}-2)^{3)}$. Interestingly, the stereospecificities of hydration by L- and D-BP

\footnotetext{
*Correspondence to: Shirou Tsuchida, Department of Molecular Biosciences, School of Pharmaceutical Sciences, Health Sciences University of Hokkaido, 1757 Kanazawa, Tobetsu-cho, Ishikari-gun, 061-0293 Hokkaido, JAPAN

E-mail: tsuchida@ hoku-iryo-u.ac.jp

Accepted December 21, 2010 (received for review November 25, 2010)

Journal of Oleo Science ISSN 1345-8957 print / ISSN 1347-3352 online

http://www.jstage.jst.go.jp/browse/jos/
} 
are different; i.e., trans-2-enoyl-CoA is converted to 3-oxoacyl-CoA via $3(S)$ - and 3 $(R)$-hydroxyacyl-CoA by Land D-BP, respectively. However, the physiological significance of these stereospecificities is not clear. In addition, previous studies on this subject have not chemically investigated the absolute configuration of the product(3-hydroxyacyl-CoA) or directly analyzed the stereospecifcity ${ }^{16-19)}$. In a previous report, we obtained enantiomeric 3-hydroxyhexadecanoic acids and determined their absolute configuration in detail ${ }^{20)}$. In this study, we investigate the development of a method for analyzing the stereospecificity of enoyl-CoA hydratase using high-performance liquid chromatography (HPLC) equipped with a chiral separation column. Moreover, we describe the effective application of this method to subcellular fractions and purified bifunctional proteins obtained from rat liver.

\section{EXPERIMENTAL PROCEDURES}

\subsection{Chemicals and instruments}

The lithium salt of CoA was purchased from Oriental Yeast (Tokyo, Japan). All other materials and solvents were of analytical grade, and deionized water was used throughout the study. Proton nuclear magnetic resonance $\left({ }^{1} \mathrm{H}-\right.$ NMR) spectrometry was recorded at $400 \mathrm{MHz}$ on a JNM-EX 400 spectrometer (JEOL Ltd., Tokyo, Japan). HPLC was carried out using a Shimadzu LC 6-A system equipped with SPD-10vp (Shimadzu Co., Kyoto, Japan); detection was performed at $260 \mathrm{~nm}$. A CHIRALPAK AD-RH $(4.6 \times 150$ mm, $5 \mu \mathrm{m}$, Daicel Chemical Ind. Ltd., Tokyo, Japan) was used as the separation column. The samples were centrifuged using a CR21 with an R22A2 angle rotor(Hitachi, Japan) or an Avanti HP-30I centrifuge with a JS-24.38 swing rotor(Beckman Coulter, Japan).

\subsection{CoA esters}

The CoA esters used in this study are shown in Fig. 1. To ensure that their correct structures were obtained, all CoA esters were synthesized and their structures confirmed via ${ }^{1} \mathrm{H}-\mathrm{NMR}$. For the synthesis of trans-2-hexadecenoyl-CoA, tetradecanal (500 mg) was first derived from tetradecanol using the Parikh-Doering oxidation ${ }^{21)}$ and then dissolved in 1,2-dichloroethane $(30 \mathrm{~mL})$ to obtained a $2.36 \mathrm{mmol} \mathrm{solu}$ tion. A stabilized phosphonium ylide, ethyl (triphenylphosphoranylidene) acetate (1.5 g) was added to the solution, which was then refluxed for $1 \mathrm{~h}$. After the reaction, the solvent was evaporated and the residue was purified using silica gel column chromatography[silica gel (50 g); hexane/ethyl acetate $=50 / 1(\mathrm{v} / \mathrm{v})]$ to produce the desired ethyl trans-2-hexadecenoate oil. The ester (284 mg, 1 $\mathrm{mmol}$ ) was then dissolved in $20 \mathrm{~mL}$ of a tert-butanol/water (1:1) solution followed by the addition of potassium tertbutoxide (1.12 g, $10 \mathrm{mmol})$ and then stirred overnight at
trans-2-hexadecenoyl-CoA
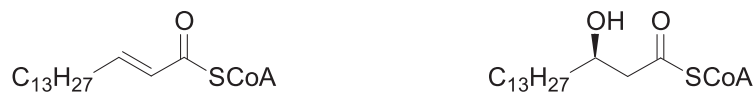

3(R)-hydroxyhexadecanoyl-CoA

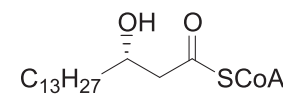

3(S)-hydroxyhexadecanoyl-CoA

Fig. 1 CoA esters used in this study. Trans-2-hexadecenoyl-CoA is a substrate for hydratases, and 3(R)- and 3(S)-hydroxyhexadecanoyl-CoA are predicted products of hydratases.

room temperature. After the reaction was complete, the mixture was acidified using $2 \mathrm{M}$ hydrochloride solution and ethyl acetate was added to extract the resultant trans2-hexadecenoic acid. The organic layer was washed with water and a saturated sodium chloride solution and dried with anhydrous sodium phosphate. The solvent was evaporated and the residue was purified using silica gel column chromatography [silica gel $(10 \mathrm{~g})$; hexane/ethyl acetate $=$ 10/1 (v/v) ] to obtain amorphous trans-2-hexadecenoic acid (170 mg, $668 \mathrm{mmol}$ ). The trans-2-hexadecenoic acid was further reacted to yield the corresponding CoA ester in accordance with a previously reported method $\left.{ }^{22}\right)$. Compounds $3(R)$ - and $3(S)$-hydroxyhexadecanoyl -CoA were synthesized as described in our previous article ${ }^{20)}$.

\subsection{Subcellular fractions and bifunctional proteins}

The light mitochondrial fraction (L-fraction) was prepared in accordance with a previous report by de Duve et $a l .{ }^{23)}$ : Approximately $10 \mathrm{~g}$ of rat liver (Wistar strain, male, 7 weeks old)was minced and homogenized with $60 \mathrm{~mL}$ of a sucrose ethylenediaminetetraacetic acid(EDTA) solution (SE; $0.25 \mathrm{M}$ sucrose; $1 \mathrm{mM}$ EDTA). The homogenate was centrifuged at $800 \times \mathrm{g}$ for $10 \mathrm{~min}$, and the supernatant was then centrifuged at 3,300 $\times \mathrm{g}$ for an additional $10 \mathrm{~min}$. Subsequently, the resultant supernatant was centrifuged at $32,000 \times \mathrm{g}$ for $10 \mathrm{~min}$. The sediment was suspended in 15 $\mathrm{mL}$ of $\mathrm{SE}$ solution and was preserved at $-80^{\circ} \mathrm{C}$ until use. This operation was carried out at $0 \sim 4{ }^{\circ} \mathrm{C}$.

Subcellular fractions were obtained by centrifuging with a modified sucrose density gradient, as was reported by Osumi et $a{ }^{24)}$. Sucrose solutions of the following volumes and concentrations were sequentially added to a rotor tube: $6 \mathrm{~mL}$ of $50.9 \%(\mathrm{w} / \mathrm{w}), 8 \mathrm{~mL}$ of $45.8 \%(\mathrm{w} / \mathrm{w}), 10 \mathrm{~mL}$ of $37.4 \%(\mathrm{w} / \mathrm{w})$, and $5 \mathrm{~mL}$ of $23.2 \%(\mathrm{w} / \mathrm{w})$ sucrose. These sucrose solutions were prepared in $50 \mathrm{mM} \operatorname{HEPES}(\mathrm{pH}$ 7.0). The L-fraction $(3 \mathrm{~mL}$ ) was then layered onto the sucrose gradient and centrifuged at $100,000 \times \mathrm{g}$ for $2 \mathrm{~h}$. The subcellular fractions $(3 \mathrm{~mL})$ were collected from the top using a perista pump and stored at $-80^{\circ} \mathrm{C}$ until use. The distribu- 
tions of mitochondria and peroxisomes were determined by measuring the succinic-cytochrome $c$ oxidase ${ }^{25)}$ and catalase $^{26)}$, respectively. L- and D-BP were prepared from rat liver in accordance with previous reports by Osumi et $a l^{27)}$ and Jiang et $a l .^{28)}$, respectively. The amount of protein was measured by a modified Lowry method using bovine serum albumin as the standard ${ }^{29)}$.

\subsection{Incubation and HPLC analysis}

A solution of $230 \mu \mathrm{L}$ of admixture $[50 \mathrm{mM}$ phosphate buffer (pH 7.5), trans-2-hexadecenoyl-CoA] and $20 \mu \mathrm{L}$ of the subcellular fraction or $20 \mu \mathrm{L}$ of the L-BP or D-BP solution was incubated at $30^{\circ} \mathrm{C}$. The concentrations of the substrate (trans-2-hexadecenoyl-CoA), amount of either L-BP or D-BP, and the time required for reaction were investigated. After incubation, the mixture was ice-cooled and 50 $\mu \mathrm{L}$ of $100 \mathrm{mM}$ hydrochloride solution was added to stop the reaction. The mixture was filtered using a Millex-LG filtering unit(Millipore, Japan), and $20 \mu \mathrm{L}$ of filtrate was injected onto an HPLC column. HPLC was carried out under the following conditions: mobile phase of $35 / 65(\mathrm{v} / \mathrm{v})$ of 50 $\mathrm{mM}$ phosphate buffer $(\mathrm{pH} 7.0) /$ methanol; flow rate of 0.5 $\mathrm{mL} / \mathrm{min}$; detection at $260 \mathrm{~nm}$; and column temperature of $25^{\circ} \mathrm{C}$. The measurements of the quantities of $3(R)$ - and 3 $(S)$-hydroxyhexadecanoyl-CoA were calibrated using an absolute calibration curve method.

\section{RESULTS AND DISCUSSION}

\subsection{Synthesis of trans-2-hexadecenoyl-CoA}

Trans-2-hexadecenoic acid was synthesized from the corresponding aldehyde (tetradecanal) via a Wittig reaction using a stabilized phosphonium ylide[ethyl (triphenylphosphoranylidene) acetate] to yield ethyl 2-hexadecenoate. The synthesis of the trans isomer was confirmed using thin-layer chromatography (TLC) and was free of the cis isomer, as assessed by ${ }^{1} \mathrm{H}-\mathrm{NMR}$. Initially, ethyl trans2-hexadecenoate was hydrolyzed using potassium hydroxide/methanol; however, the methoxide ion attacked the double bond to form an undesired compound (data not shown). Therefore, potassium tert-butoxide/tert-butanol, which features higher steric bulk, was used to generate the desired trans-2-hexadecenoic acid. The characterization data of trans-2- hexadecenoic acid were as follows: ${ }^{1} \mathrm{H}-$ $\mathrm{NMR}\left(\mathrm{CDCl}_{3}, \mathrm{ppm}\right): 0.88(3 \mathrm{H}, \mathrm{t}, J=7.0 \mathrm{~Hz}, 16-\mathrm{H}), 1.26(2 \mathrm{H}$, m, 6-H, 7-H, 8-H. 9-H, 10-H, 11-H, 12-H, 13-H, 14-H, 15-H), $1.46(2 \mathrm{H}, \mathrm{m}, 5-\mathrm{H}), 2.22(2 \mathrm{H}, \mathrm{m}, 4-\mathrm{H}), 5.80(1 \mathrm{H}, \mathrm{d}, J=15.6$ $\mathrm{Hz}, 2-\mathrm{H}), 7.08$ (1H, sext, $J=15.8 \mathrm{~Hz}, 7.0 \mathrm{~Hz}, 3-\mathrm{H}), \mathrm{MS}(\mathrm{EI})$ : $\mathrm{m} / \mathrm{z} 254\left(\mathrm{M}^{+}\right), 236\left(\mathrm{M}^{+}-\mathrm{H}_{2} \mathrm{O}\right)$. The trans double bond was detected as signals at $5.80 \mathrm{~Hz}$ and $7.08 \mathrm{~Hz}$, corresponding to $2-\mathrm{H}$ and $3-\mathrm{H}$, respectively. If the cis-form, the signals corresponding to $2-\mathrm{H}$ and $3-\mathrm{H}$ were observed at $5.94 \mathrm{~Hz}$ and $6.41 \mathrm{~Hz}$, respectively. As these signals were not ob- served in the synthesized product, we can confirm the purity of the trans product. After confirmation of the structure, trans-2-hexadecenoic acid was further reacted to form its corresponding CoA ester.

\subsection{Investigation of HPLC conditions}

Compounds $3(R)$ - and 3 $(S)$-hydroxyhexadecanoyl-CoA were detected by monitoring the absorbance at $260 \mathrm{~nm}$, which is the maximum absorbance (Fig. 2A). The absorbance of 3-hydroxyhexadecanoyl-CoA showed the same pattern throughout the $\mathrm{pH}$ range of $4.0 \sim 9.0$ (data not shown).

The effect of the organic solvent on the separation of 3 $(R)$ - and 3 $(S)$-hydroxyhexadecanoyl-CoA was investigated using reverse-phase chiral columns, because CoA esters
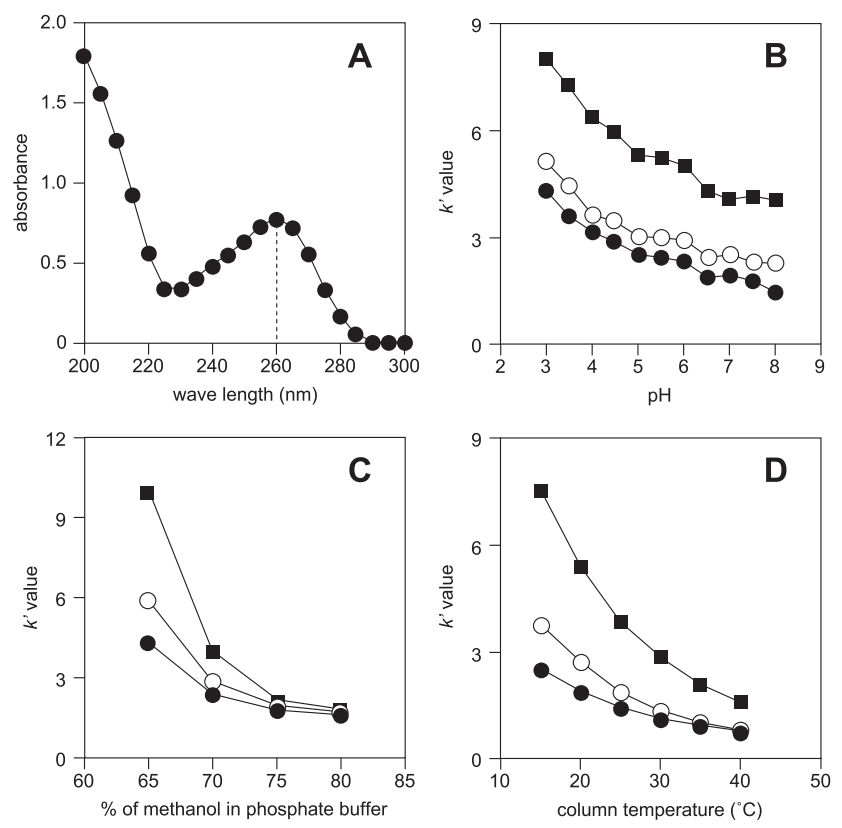

Fig. 2 Absorbance of 3-hydroxyhexadecanoyl-CoA at $\mathrm{pH} 7.0$ (A), and the effects of $\mathrm{pH}(\mathrm{B})$, proportion of methanol in mobile phase $(\mathrm{C})$, and column temperature (D) on the $k^{\prime}$ value.

Procedures B-D were performed under the following conditions: flow rate of $0.5 \mathrm{~mL} / \mathrm{min}$; mobile phase of $50 \mathrm{mM}$ phosphate buffer ( $\mathrm{pH} 5.0$, except for B)/methanol = 35/65 (v/v, except for $\mathrm{C})$; detection at $260 \mathrm{~nm}$; and column temperature of $25^{\circ} \mathrm{C}$ (except for D). The $k$ ' value was calculated according to the following formula: $k^{\prime}=\left(t_{\mathrm{R}}-t_{0}\right) / t_{0}$, where $t_{\mathrm{R}}$ is the retention time of each CoA ester. The void volume $\left(t_{0}\right)$ was measured using sodium nitrate at $210 \mathrm{~nm}$. , 3(R)hydroxyhexadecanoyl-CoA; $\bigcirc, 3(S)$-hydroxyhexadecanoyl-CoA; $\boldsymbol{\square}$, trans-2-hexadecenoylCoA. 


\section{S. Tsuchida, K. Kawamoto, K. Nunome et al.}

are amphiphilic compounds. Two kinds of chiral separation columns using either amylose (CHIRALPAK AD-RH) or cellulose (CHIRALPAK OD-RH) derivatives as the stationary phase were examined. Both water/acetonitrile and water/ methanol solutions were compared using theses columns. When CHIRALPAK AD-RH was used, the tops of the distorted peaks corresponding to $3(R)$ - and $3(S)$-hydroxyhexadecanoyl-CoA were separated with water/methanol = $50 / 50 \sim 40 / 60(\mathrm{v} / \mathrm{v})$. Peaks corresponding to $3(R)-$ and 3 $(S)$-hydroxyhexadecanoyl-CoA overlapped completely with water/acetonitrile $=90 / 10 \sim 60 / 40(\mathrm{v} / \mathrm{v})$. Therefore, the water/methanol system is more suitable for the separation of $3(R)$ - and $3(S)$-hydroxyhexadecanoyl-CoA than the water/acetonitrile system. When CHIRALPAK OD-RH was used, $3(R)$ - and 3 $(S)$ - hydroxyhexadecanoyl-CoA were not separated at all, regardless of the ratio or identity of the organic solvents. Accordingly, CHIRALPAK AD-RH was used in the following studies. However, if the separation conditions had been examined in detail, CHIRALPAK OD$\mathrm{RH}$ would have been used for this study.

Next, the effect of the $\mathrm{pH}(\mathrm{pH} 3.0 \sim 8.0)$ of the mobile phase on the $k$ ' value was investigated using $50 \mathrm{mM}$ phosphate buffer/methanol $=35 / 65(\mathrm{v} / \mathrm{v})($ Fig. 2B) . The CoA esters have three phosphate groups. Therefore, the $\mathrm{pH}$ should affect their retention; specifically, the retention of the CoA esters onto the reverse-stationary phase should increase at lower $\mathrm{pH}$ levels and decreased at higher $\mathrm{pH}$ levels. Accordingly, the $k$ ' value increased in the $\mathrm{pH}$ range of $3.0 \sim 4.0$ and the peaks were broad and showed tailing. However, the $k$ ' value did not change significantly in the $\mathrm{pH}$ range of $5.0 \sim 8.0$. The mobile phase adjusted to $\mathrm{pH} 5.0$ separated the CoA esters satisfactorily and eluted all CoA esters within $30 \mathrm{~min}$.

To optimize the separation conditions, the effect of the proportion $(\%, v / v)$ of methanol in the mobile phase on the separation of the CoA esters was investigated within the range of $60 \sim 80 \%(\mathrm{v} / \mathrm{v})$ (Fig. 2C). The $k$ ' values of the CoA esters were extremely increased at less than $60 \%(\mathrm{v} / \mathrm{v})$, and the CoA ester peaks overlapped at $75 \sim 80 \%(\mathrm{v} / \mathrm{v})$. The CoA esters were separated satisfactorily at $65 \%(\mathrm{v} / \mathrm{v})$. The buffer/methanol ratio of $35 / 65(\mathrm{v} / \mathrm{v})$ was found to be the most suitable for the separation of the CoA esters.

However, satisfactory reproducibility was not obtained with the above conditions. Finally, it was determined that the column temperature affected the $k^{\prime}$ value (Fig. 2D); i. e., the $k^{\prime}$ values increased as the column temperature decreased. The peaks corresponding to $3(S)$-hydroxyhexadecanoyl-CoA and trans-2-hexadecenoyl-CoA showed tailing at $15^{\circ} \mathrm{C}$, whereas the $3(R)$ - and $3(S)$-hydroxyhexadecanoyl-CoA were not separated at $35 \sim 40^{\circ} \mathrm{C}$. The separation of the CoA esters was optimized at $25^{\circ} \mathrm{C}$. Therefore, it was determined that a constant column temperature was very important for reproducibility. This is probably because the temperature affects the motion of the alkyl
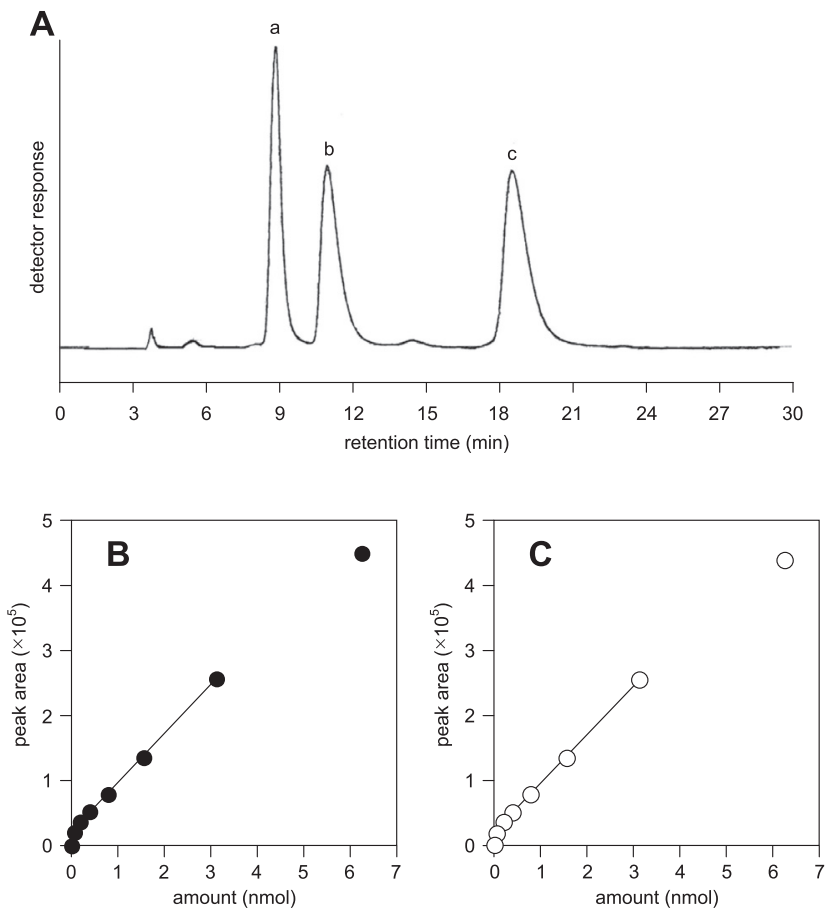

Fig. 3 Standard chromatograms of CoA esters (A): a, 3(R)- hydroxyhexadecanoyl-CoA; b, 3(S)-hydroxyhexadecanoyl-CoA; and c, trans-2-hexadecenoyl-CoA.

Calibration curves for 3(R)- and 3(S)-hydroxyhexadecanoyl-CoA are shown in $(\mathrm{B})$ and $(\mathrm{C})$, respectively.

group of the CoA ester, which affects the affinity between the CoA esters and the stationary phase.

After considering all factors, the optimized conditions for the separation of CoA esters using HPLC were determined, as described above (see Experimental section). The $k$ values of $3(R)$ - and $3(S)$-hydroxyhexadecanoyl-CoA and trans-2-hexadecenoyl-CoA were 1.31, 1.85, and 3.53, respectively. The separation factors $(\alpha)$ between $3(R)$ - and $3(S)$-hydroxyhexadecanoyl-CoA and between $3(S)$ - hydroxyhexadecanoyl-CoA and trans-2-hexadecenoyl-CoA were 1.41 and 1.91, respectively. A standard chromatogram of the CoA esters is shown in Fig. 3A. The limits of detection of the CoA esters were $5 \mathrm{pmol}$ (signal-to-noise ratio= 6 ) and the limits of quantification were estimated to be 15 pmol. Good linearity was obtained over the range of 50 pmol-3 nmol with correlation coefficients of $>0.999$. The calibration curves for $3(R)$ - and $3(S)$-hydroxyhexadecanoyl-CoA are shown in Fig. 3B and 3C, respectively.

\subsection{Enoyl-CoA hydratase assay using subcellular fractions}

The succinic-cytochrome c reductase activity and the catalase activity of each fraction were measured. Fraction 6 had the highest succinic-cytochrome c reductase activity, 
and fraction 9 had the highest catalase activity (Fig. 4A). The developed method for the concurrent quantification of $3(R)$ - and 3 $(S)$-hydroxyhexadecanoyl-CoA was tested using subcellular fractions obtained from rat liver homogenate. The enoyl-CoA hydratase activity varied in all fractions. In particular, fractions 6 $\sim 9$ showed higher enoylCoA hydratase activity. In addition, both $R$ - and $S$-specific activities were observed (Fig. 4B). It is thought that the peroxisomal enoyl-CoA hydratases were mixed in the mitochondrial fraction because the monolayer membrane of peroxisome was broken when the tissue was homogenized. Furuta et al. reported that mitochondrial enoyl-CoA hydratase is heat stable and L-specific ${ }^{30)}$. Accordingly, each subcellular fraction was heat treated $\left(60^{\circ} \mathrm{C}, 2 \mathrm{~min}\right)$ and used for incubation. The stereospecific enoyl-CoA hydratase activi-

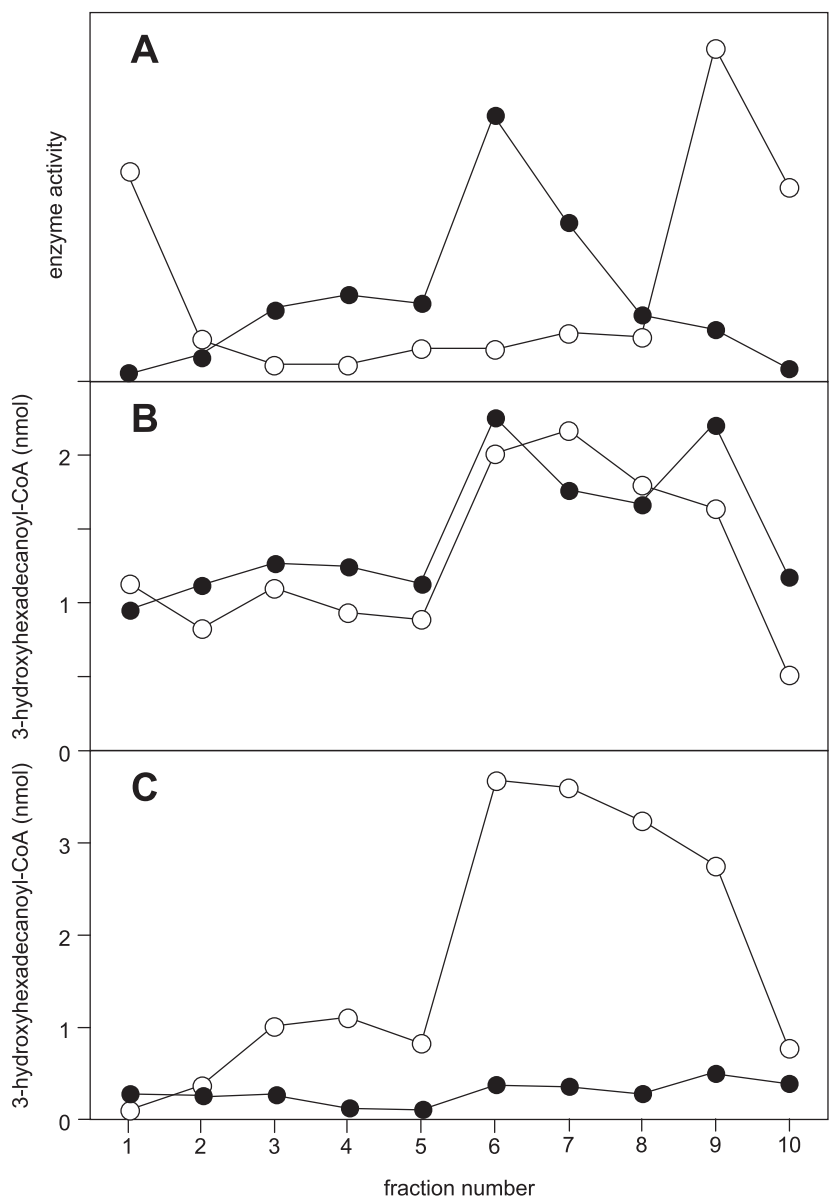

Fig. 4 Sucrose density gradient centrifugation of the light mitochondrial fraction (L-fraction).

Distribution of activities of marker enzymes (A): $\bigcirc$, succinic- cytochrome $c$ reductase; $\bigcirc$, catalase. Distribution of enoyl-CoA hydratase before (B) and after the heat-treatment (C): $\mathrm{O}$, $3(R)$ - hydroxyhexadecanoyl-CoA; $\bigcirc, 3(S)$ hydroxyhexadecanoyl-CoA.
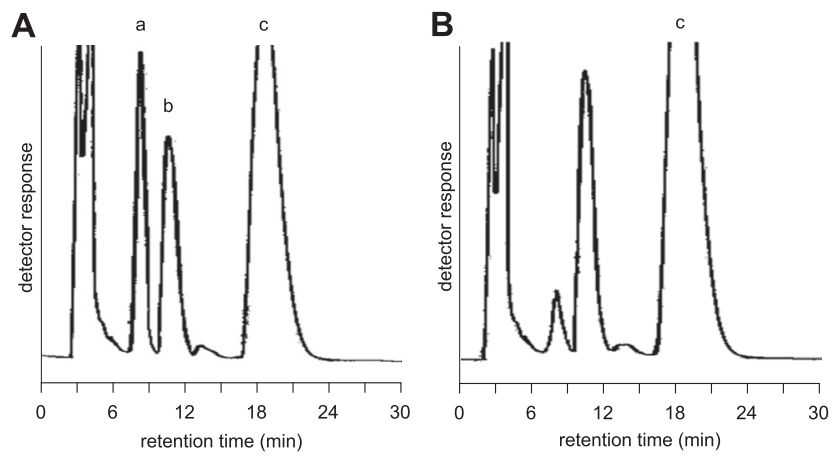

Fig. 5 Chromatograms of products obtained after incubation of trans-2-hexadecenoyl-CoA with fraction 6 (A) and with heat-treated fraction 6 (B). a, 3(R)-hydroxyhexadecanoyl-CoA; b, 3(S)hydroxyhexadecanoyl-CoA; c, trans-2-hexadecenoyl-CoA (substrate).

ties after the heat treatment of all subcellular fractions are shown in Fig. 4C. The production of $3(R)$-hydroxyhexadecanoyl-CoA was remarkably decreased after heat treatment in all fractions, although the production of 3 $(S)$-hydroxyhexadecanoyl-CoA remained stable. In the chromatograms of trans-2-hexadecenoyl-CoA with fraction 6 , peaks corresponding to both $3(S)$ - and $3(R)$-hydroxyhexadecanoyl-CoA were observed (Fig. 5A). When fraction 6 was heat treated at $60^{\circ} \mathrm{C}$ for 2 min prior to incubation, the peaks corresponding to $3(R)$ - hydroxyhexadecanoylCoA decreased, although the peaks of $3(S)$ - hydroxyhexadecanoyl-CoA did not change (Fig. 5B). From these results, it was directly confirmed that the heat-stable hydratase has S-specificity.

\subsection{Enoyl-CoA hydratase assay using L- and D-BP obtained from rat liver}

The developed method was applied to the analysis of Land D-BP. In the HPLC chromatograms, L-BP produced 3 $(S)$-hydroxyhexadecanoyl- CoA from trans-2-hexadecenoyl-CoA (Fig. 6A). In contrast, D-BP produced 3 $(R)$-hydroxyhexadecanoyl-CoA from trans-2-hexadecenoyl- CoA (Fig. 6B). Before the measurement of the L- and D-BP activities, the incubation conditions were examined. Accordingly, the substrate concentration was set to $0.2 \mathrm{mM}$ (Fig. 7A), amount of L-BP or D-BP used were set to $0.3 \mu \mathrm{g}$ (Fig. 7B), and incubation time was set to $20 \mathrm{~min}$ (Fig. 7C). The enzymatic activities of L- and D-BP were $243.7 \pm 14.1 \mathrm{nmol} /$ $\mathrm{min} / \mathrm{mg}$ and $993.6 \pm 22.3 \mathrm{nmol} / \mathrm{min} / \mathrm{mg}$, respectively (Table 1). Although an accurate comparison is difficult because of the different of substrates, purification methods, and incubation conditions, theses values are lower than those previously reported $^{27,28,30-33)}$.

Peaks corresponding to $3(R)$ - and $3(S)$-hydroxyhexadecanoyl-CoA were observed in HPLC chromatograms of 

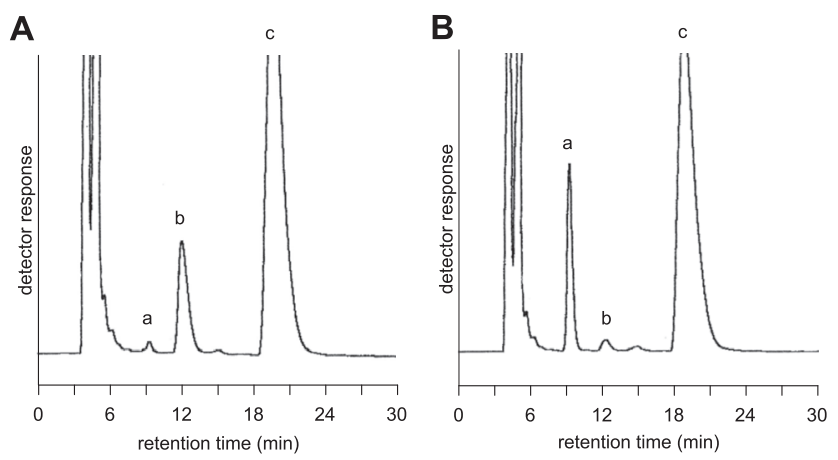

Fig. 6 Chromatograms of products obtained after incubation of trans-2-hexadecenoyl-CoA with L-BP (A) and D-BP (B).

a, 3(R)-hydroxyhexadecanoyl-CoA; b, 3(S)hydroxyhexadecanoyl-CoA; c, trans-2-hexadecenoyl-CoA (substrate).

Fig. $6 \mathrm{~A}$ and $6 \mathrm{~B}$, respectively. These peaks are below the quantification limit. It was determined that both enzyme solutions were not pure enough and were likely contaminated with another hydratase. Increased purification of the enzymes is needed to accurately compare the activities of L- and D-BP. However, through the investigation of L- and D-BP obtained from rat liver by this method, it was directly confirmed that the hydratase activities of L- and D-BP are $S$ - and $R$-specific, respectively.

In this study, an analytical method for the investigation of the stereospecificity of enoyl-CoA hydratases was established. In addition, this method was applied to subcellular fractions and purified bifunctional proteins. The key advantage of this method is the concurrent quantification of 3 $(R)$ - and $3(S)$-hydroxyacyl-CoA, which was shown to be useful for the rigorous analysis of the stereospecificity of enoyl-CoA hydratases. In addition, this method does not need pre- or post-labeling for the determination of the absolute configuration of 3-hydroxy group ${ }^{34)}$. The advantages of this method enable a simple diagnosis of a peroxisomal disorder because $3(R)$-hydroxyacyl-CoA is a specific intermediate of peroxisomal $\beta$-oxidation. In addition, if a fundamental therapy for D-BP deficiency is established in the future, (e.g., gene therapy), this analytical method will be useful for the assessment of therapeutic effects.
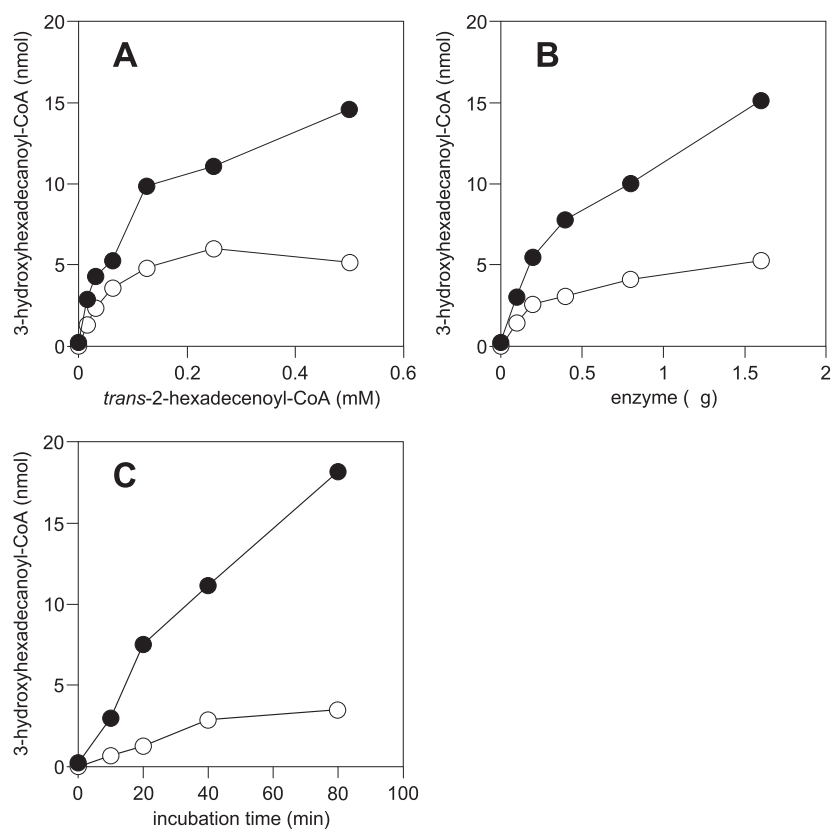

Fig. 7 Examination of incubation conditions for L- and D-BP. Effect of substrate (trans-2-hexadecenoyl-CoA) concentration (A), amount of enzymes (B), and incubation time (C) on the formation of 3(R)- and 3(S)-hydorxyhexadecanoyl-CoAs.

Incubation was carried out under the following conditions: $230 \mu \mathrm{L}$ of admixture $[50 \mathrm{mM}$ phosphate buffer ( $\mathrm{pH} 7.5$ ), $0.2 \mathrm{mM}$ (except for A) trans-2-hexadecenoyl-CoA] was added to $20 \mu \mathrm{L}$ (15 $\mu \mathrm{g} / \mathrm{mL}$, except for B) of L-BP or D-BP solution, and incubated at $30^{\circ} \mathrm{C}$ for $20 \mathrm{~min}$ (except for $\mathrm{C})$. 3(S)-hydroxyhexa- decanoyl-CoA.

\section{References}

1) Hashimoto, T. Peroxisomal $\beta$-oxidation enzymes. Cell Biophys. 32, 63-72(2000).

2) Reddy, J. K.; Hashimoto, T. Peroxisomal $\beta$-oxidation and peroxisome proliferator-activated receptor alpha: an adaptive metabolic system. Annu. Rev. Nur. 21, 193-230 (2001).

3) Poirier, Y.; Antonenkov, V. D.; Glumoff, T.; Hiltunen, J.K. Peroxisomal $\beta$-oxidation- a metabolic pathway

Table 1

\begin{tabular}{ccc}
\hline \multirow{2}{*}{ enzyme } & \multicolumn{2}{c}{ formation of 3-hydroxyhexadecanoyl-CoA $(\mathrm{nmol} / \mathrm{min} / \mathrm{mg})^{\mathrm{a}}$} \\
\cline { 2 - 3 } & 3 (S)-hydroxyhexadecanoyl-CoA & 3(R)-hydroxyhexadecanoyl-CoA \\
\hline L-BP & $243.7 \pm 14.1$ & $-{ }^{\mathrm{b}}$ \\
D-BP & $-{ }^{\mathrm{b}}$ & $993.6 \pm 22.3$ \\
\hline
\end{tabular}

${ }^{\mathrm{a}}$ Mean \pm S.D. $(\mathrm{n}=3)$

${ }^{\mathrm{b}}$ less than limit of quantification 


\section{HPLC Analysis of Stereospecificity of Enoyl-CoA Hydratase}

with multiple functions. Biochem. Biophys. Acta 1736, 1413-1426(2006).

4) Poulos, A. Very long chain fatty acids in higher animals -a review. Lipids 30, 1-14(1995).

5) Mukherji, M.; Schofield, C. J.; Wierzbicki, A. S.; Wanders, R. J.; Lloyd, M. D. The chemical biology of branched-chain lipid metabolism. Prog. Lipid Res. 42, 359-376 (2003).

6) Verhoeven, N. M.; Jakobs, C. Human metabolism of phytanic acid and pristanic acid. Prog. Lipid Res. 40, 453-66 (2001).

7) Ferdinandusse, S.; Houten, S. M. Peroxisomes and bile acid biosynthesis. Biochim. Biophys. Acta 1736, 1427-1440 (2006)

8) Wanders, R. J. Peroxisomes, lipid metabolism, and human disease. Cell Biochem. Biophys. 32, 89-106 (2000).

9) Clayton, P. T. Clinical consequences of defects in peroxisomal $\beta$-oxidation. Biochem. Soc. Trans. 29, 298-305 (2001).

10) Brosius, U.; Gärtner, J. Cellular and molecular aspects of Zellweger syndrome and other peroxisome biogenesis disorders. Cell Mol. Life Sci. 59, 1058-1069(2002).

11) Wanders, R. J. Peroxisomes, lipid metabolism, and peroxisomal disorders. Mol. Gene Metab. 83, 16-27 (2004).

12) Wanders, R. J.; Waterham, H. R. Biochemistry of mammalian peroxisomes revisited. Annu. Rev. Biochem. 75, 295-332(2006)

13) Wanders, R. J.; Waterham, H. R. Peroxisomal disorders: the single peroxisomal enzyme deficiencies. Biochim. Biophys. Acta 1763, 1707-1720(2006).

14) Shimozawa, N. J. Molecular and clinical aspects of peroxisomal diseases. Inherit. Metab. Dis. 30, 193-197 (2007).

15) Wnaders, R. J.; Ferdinanduss, S.; Brites, P.; Kemp, S. Peroxisomes, lipid metabolism and lipotoxicity. Biochim. Biophys. Acta 1801, 272-280 (2010).

16) Kobayashi, A.; Jiang, L. L.; Hashimoto, T. Two mitochondrial 3-hydroxyacyl-CoA dehydrogenase in bovine liver. J. Biochem. 119, 775-782(1996).

17) Jin, S. J.; Hoppel, C. L.; Tserng, K.Y . Incomplete fatty acid oxidation. The production and epimerization of 3-hydroxy fatty acids. J. Biochem. 267, 119-125 (1992).

18) Dieuaide-Noubani, M.; Novikov, D.; Baumgart, E.; Vanhooren, J. C.; Fransen, M.; Goethals, M.; Vandekerckhove, J.; Van, Veldhoven, P. P.; Mannaerts, G. P. Further characterization of the peroxisomal 3-hydroxyacyl-CoA dehydrogenase from rat liver. Relationship between the different dehydrogenases and evidence that fatty acids and the $\mathrm{C} 27$ bile acids di- and tri-hydroxycoprostanic acids are metabolized by separate multifunctional proteins. Eur. J. Biochem. 240 ,
660-666 (1996).

19) Dieuaide-Noubhani, M.; Asselberghs, S.; Mannaerts, G. P.; Van Veldhoven, P. P. Evidence that multifunctional protein 2 , and not multifunctional protein 1 , is involved in the peroxisomal $\beta$ - oxidation of pristanic acid. Biochem. J. 325, 367-373(1997).

20) Tsuchida, S.; Kawamoto, K.; Nunome, K.; Hamaue, N.; Hui, S. P.; Murai, T.; Aoki, T.; Kurosawa, T. Chiral separation, determination of absolute configuration, and high-performance liquid chromatography detection of enantiomeric 3-hydroxyhexadecanoyl- CoA. J. Oleo sci. 60, 87-92 (2011).

21) Parikh, J. P.; Doering, W. E. Sulfur trioxide in the oxidation of alcohols by dimethyl sulfoxide. J. Am. Chem. Soc. 84, 5505-5507(1967).

22) Kurosawa, T.; Sato, M.; Inoue, K.; Yoshimura, T.; Tohma, M.; Jiang, L. L.; Hashimoto, T. Separation of stereoisomers of $\mathrm{C}_{27}$-bile acid CoA esters by liquid chromatography and its application to the study of the stereospecificities of D- and L-bifunctional proteins in bile acid biosynthesis. Anal. Chim. Acta 365, 249-257 (1998).

23) de Duve, C.; Pressman, B. C., Gianetto, R.; Wattiaux, R.; Applemans, F. Tissue fractionation studies. 6. Intercellular distribution patterns of enzymes in rat liver tissue. Biochem. J. 60, 604-617(1955).

24) Osumi, T.; Hashimoto, T.; Enhancement of fatty acylCoA oxidizing activity in rat liver peroxisomes by di(2-methyl) phtalate. J. Biochem. (Tokyo). 83, 1361-1365(1978).

25) Polakis, E. S.; Bartley, W.; Meek, G. A. Change in the structure and enzyme activity of Saccharomyces cerevisiae inresponse to change in environment. Biochem. J. 83, 1361-1365 (1964).

26) Aebi, H., Measurement of catalase reaction in Methods of Enzymatic Analysis. $2^{\text {nd }}$ ed. (Bergmeyer, H.U. ed), Verlag Chemie Wienheim pp. 673-684(1974).

27) Osumi, T.; Hashimoto, T. Peroxisomal $\beta$-oxidation system of rat liver. Copurification of enoyl-CoA hydratase and 3-hydroxyacyl- CoA dehydrogenase. Biochem. Biophys. Res. Commun. 89, 580-584(1979).

28) Jiang, L. L.; Miyazawa, S.; Hashimoto, T. Purification and properties of rat D-3-hydroxyacyl-CoA dehydrates/D-3- hydroxyacyl-CoA dehydrogenase bifunctional protein. J. Biochem. 120, 633-641 (1996) .

29) Lowry, O. H.; Rosebrough, N. J.; Farr, A. L.; Randall, R. J. Protein measurement with the Folin phenol reagent. J. Biol. Chem. 193, 265-275(1951).

30) Furuta, S.; Miyazawa, S.; Osumi, T.; Hashimoto, T. Properties of mitochondrial and peroxisomal enoylCoA hydratases from rat liver. J. Biochem. 88, 1059-1070 (1980).

31) Palosaari, P. M.; Hiltunen, J. K. Peroxisomal bifunctional protein from rat liver is a trifunctional enzyme 


\section{S. Tsuchida, K. Kawamoto, K. Nunome et al.}

possessing 2-enoyl-CoA hydratase, 3-hydroxyacyl-CoA dehydrogenase, and $\Delta^{3}, \Delta^{2}$-enoyl- CoA isomerase activities. J. Biol. Chem. 265, 2446-2449 (1990).

32) Jiang, L. L.; Kurosawa, T.; Sato, M.; Suzuki, Y.; Hashimoto, T. Physiological role of D-3-hydroxyacyl-CoA dehydratase/D-3- hydroxyacyl-CoA dehydrogenase bifunctional protein. J. Biochem. 121, 506-513(1997).

33) Qin, Y. M.; Poutanen, M. H.; Helander, H. M.; Kvist, A.-P.; Siivari, K. M.; Schmitz, W.; Conzelmann, E.; Hell- man, U.; Hiltunen, J. K. Peroxisomal multifunctional enzyme of $\beta$-oxidation metabolizing D-3-hydroxyacylCoA esters in rat liver: molecular cloning, expression and characterization. Biochem. J. 321, 21-28(1997).

34) Hammarström, S.; Hamberg, M. Steric analysis of 3-, $\omega 4-, \omega 3-$ and $\omega 2$-hydroxy acids and various alkanols by gas-liquid chromatography. Anal. Biochem. 52, 169-179 (1973). 